\title{
VEGF Gene Transfer for Diabetic Polyneuropathy: A Randomized Double Blinded Trial
}

\author{
Allan H. Ropper ${ }^{1}$, Kenneth C. Gorson ${ }^{2}$, Clifton L. Gooch ${ }^{3}$, David H. Weinberg ${ }^{2}$, Ann \\ Pieczek $^{2}$, James H. Ware ${ }^{4}$, Joshua Kershen ${ }^{5}$, Adam Rogers ${ }^{6}$, Drasko Simovic ${ }^{2}$, Peter \\ Schratzberger $^{7}$, Rudolf Kirchmair ${ }^{7}$, and Douglas Losordo ${ }^{8}$ \\ ${ }^{1}$ Department of Neurology, Brigham and Women's Hospital, Boston \\ ${ }^{2}$ Department of Neurology, St. Elizabeth's Medical Center, Boston \\ ${ }^{3}$ Department of Neurology, University of South Florida College of Medicine \\ 4 Department of Biostatistics, Harvard School of Public Health, Boston \\ ${ }^{5}$ Department of Neurology, Tufts Medical Center, Boston \\ ${ }^{6}$ Department of Ophthalmology, Tufts Medical Center, Boston \\ 7 Department of Internal Medicine, University Hospital Innsbruck, Innsbruck \\ ${ }^{8}$ Northwestern University Feinberg School of Medicine
}

\section{Abstract}

Objective-Phase 2 randomized trial of intramuscular gene transfer using plasmid vascular endothelial growth factor (VEGF) to treat diabetic polyneuropathy.

\begin{abstract}
Methods-Diabetics with polyneuropathy and no neoplasm or active retinopathy were randomized to receive VEGF:placebo 3:1. Three sets of injections were given at 8 standardized sites adjacent to the sciatic, peroneal and tibial nerves of one leg. Primary outcomes were change in symptom score at 6 months and a prespecified overall clinical and electrophysiologic improvement score. Secondary outcomes were differences in symptoms, examination scores, visual analog pain scale (VAS), nerve conduction and quantitative sensory testing.

Results-39 patients received plasmid VEGF and 11, placebo. Mean symptom score improved in both legs at 6 months, favoring VEGF over placebo $(-1.2 \pm 0.5$ vs. $-0.9 \pm 0.5 ; p<0.01$ after adjustment for change in the untreated leg) and compared to the untreated leg $(-0.7 \pm 0.5, \mathrm{p}=0.02)$. The region of sensory loss and VAS improved in the treated group ( -1.5 vs. $-0.5, \mathrm{p}=0.01) .12$ of 39 VEGF vs. 2 of 11 placebo patients met criterion for overall improvement. Other measures including nerve conduction potentials did not improve. There were 84 adverse events in VEGF patients, 22 serious and 51 events in placebo patients, 2 serious.
\end{abstract}

Direct correspondence to Dr. Ropper, aropper@partners.org, Department of Neurology, Brigham and Women's Hospital, 75 Francis St., Boston, MA 02115.

Trial registration: NCT00056290 www.clinicaltrials.gov

COI: None of the authors have a conflict of interest to report in relation to the conduct of this study. 
Interpretation-Intramuscular plasmid VEGF gene transfer improved diabetic neuropathic symptoms, meeting primary endpoint criteria for efficacy, but not affecting most secondary measures. Treatment was associated with a higher rate of serious adverse events that did not reach statistical significance. These results are not conclusive but may justify further clinical study.

\section{Introduction}

A predominantly sensory polyneuropathy is present in $7 \%$ of diabetics at the time of diagnosis and affects over $50 \%$ after 25 years of disease. ${ }^{1}$ The consequences of diabetic polyneuropathy include pain, numbness, imbalance and a predisposition to foot ulceration, the last of which is facilitated by autonomic and vascular changes as a result of neuropathy. Painless ulcerations are often unrecognized by the patient for long periods and lead to infection and toe or foot amputation at a rate 15 times higher in diabetics compared to nondiabetics.

Preclinical studies from our and other laboratories have demonstrated improved sensory behavioral features (tail flick and paw withdrawal), nerve vascularization, sciatic nerve blood flow by laser-Doppler measurement, and nerve conduction studies in diabetic animals treated with intramuscular (IM) injections of plasmid DNA encoding the vascular endothelial growth factor (VEGF) gene. ${ }^{2}$ Two other models have given similar results. ${ }^{34}$ In addition, clinical studies have indicated that the signs and symptoms of local neuropathy in patients with lower extremity critical limb ischemia improve with intramuscular injection of VEGF gene. ${ }^{5}$ These findings implicate microvascular ischemia as an important and possibly principal cause of diabetic polyneuropathy and suggest that angiogenic growth factors are a potential treatment.

Gene transfer consists of the introduction of genetic material into somatic cells in order to achieve gene expression. Of the three methods of introducing this material; viral vector, liposome DNA, and naked plasmid DNA; the last avoids the risks of viral exposure and of persistent uncontrolled expression. Although transfection efficiency is low with plasmid DNA (fewer than $1 \%$ of cells), it is highly site specific and leads to local levels of protein that are biologically active and have therapeutic effects both in vitro ${ }^{6}$ and in vivo ${ }^{7}$ without inciting a host response. Furthermore, ischemic tissues show transfection efficiencies that are fivefold higher than in normal tissue.

We conducted a randomized phase 2 trial of VEGF gene transfer for the treatment of symptomatic diabetic polyneuropathy. One leg was injected with active agent or placebo; the contralateral leg was not injected and served as an additional control.

\section{Methods}

\section{Patient recruitment and selection}

Diabetic patients with pain or numbness in the feet and legs were solicited by print and radio advertising in the Boston and New York metropolitan areas and from the diabetes clinics of our hospitals. Respondents were screened through phone calls and by review of medical records by a specially trained nurse (AP). Those with established diabetes who were taking 
oral or insulin therapy, had symptoms of polyneuropathy, and reported being free of cancer and active diabetic retinopathy were invited for screening. Those who fulfilled the study's entry criteria and had no contraindications for gene therapy (appendix A), signed informed consent for further testing and for gene therapy administration. They underwent a general physical, neurological, fundoscopic, and standard laboratory examinations and had screening for cancer (appendix B), alternative causes of polyneuropathy (appendix C), and nerve conduction studies of the peroneal, tibial and sural nerves in both legs.

The study was initiated at St. Elizabeth's Medical Center, Boston and performed at this institution and at Columbia University Medical Center, New York. The conduct of the study and informed consent methods were approved by the IRBs of the two institutions as well as the RAC and data safety monitoring board established by the sponsor, NHLBI.

\section{Study Measures}

Patients were assessed by neurologists who were blinded to treatment assignment and to the patient's laboratory data. For all scores, higher values indicate more severe involvement. Therefore, a greater decline in comparison to baseline or to the opposite leg indicates a better outcome. The symptom score (SS) encompassed 5 neuropathy related features in each leg: 1) distal leg weakness, 2) proximal leg weakness, 3) numbness, 4) paresthesias, and 5) pain (excluding at the site of skin ulceration, pain from vascular claudication or ischemic rest pain). Each symptom was graded from $0-3(0=$ none; $1=$ mild; $2=$ moderate; $3=$ severe $)$. The maximum possible SS (indicating most severe symptoms) was thus 15 (a score over 4 was required for study entry).

Lower extremity sensory testing was graded by a sensory examination score (SES), which evaluated: 1) sensory deficit for pin prick and light touch when compared to a proximal, normal region, graded $0-4(0=$ normal; $1=>75 \%$ of normal; $2=50-74 \% ; 3=25-49 \% ; 4=<25 \%)$; 2) distribution of sensory symptoms for light touch and pinprick: graded $0-4(0=$ normal; $1=$ abnormal to toes; $2=$ to ankle; $3=$ to mid-calf; $4=$ =above mid-calf); 3 ) vibration sense at the toes and ankle, graded 0-4 (0-normal; $1=$ mild loss; $2=$ moderate; $3=$ severe; $4=\mathrm{absent}$ ); and 4 ) proprioception at the great toe ( 6 trials), graded $0-4(0=6 / 6$ correct; $1=4-5 / 6 ; 2=3 / 6 ; 3=1-2 / 6$; 4=0/6). The maximum possible SES was 28 .

The motor examination score (MES) evaluated strength in both proximal and distal leg muscles using a 0-4 scale (reverse of the Medical Research Council [MRC]) scale of 1-5, as higher scores indicate more weakness). Proximal muscles tested were the iliopsoas, quadriceps and hamstrings; distal were tibialis anterior, gastrocnemius, and extensor hallucis longus/brevis. Reflex score (RS) assessed deep tendon reflexes at the knees and ankles, graded $0-4(0=$ normal, $2=$ reduced, $4=$ absent $)$; a score of 2 or more at the ankles was required as an entry criterion).

A total examination score (TES) was calculated as the sum of SES, MES, and RS. The maximum possible TES, indicative of the most severe impairment, was 64. Examination scores and nerve conduction studies were obtained within 4 weeks prior to the first injection and at 12, 24 and 52 weeks; quantitative sensory testing was performed in both legs prior to treatment and at 6 months. Ankle-brachial index, Rutherford vascular scores and fundoscopy 
with retinal photographs to screen for active proliferative diabetic retinopathy were obtained prior to treatment and at 12,24 and 52 weeks.

This scoring system and the electrophysiologic measures described below were used in our previous study of ischemic limb neuropathy and are adopted from scales that are incorporated into the NIS-LL, which was devised for quantifying the symptoms and deficits of diabetic neuropathy, ${ }^{8}$ INCAT criteria sensory sum score, ${ }^{9}$ Average Muscle Score, ${ }^{10}$ and NTSS- ${ }^{11}$ that have been used in other therapeutic trials for peripheral neuropathy. ${ }^{12}$

Standardized nerve conduction studies of the tibial and peroneal motor nerves and the sural sensory nerve were performed in both legs. Quantitative sensory testing was performed using a CASE IV (Computer Aided Sensory Evaluator; WR Medical Electronics Co., Stillwater, MN) to determine thresholds for vibration and cold sensation in the legs using the "4-2-1" algorithm. Vibration testing used calibrated $125 \mathrm{~Hz}$ mechanical oscillations in 25 graded steps from 0.1 to $576 \mathrm{um}$. One trial each for vibration and cold threshold was performed using the 4-2-1 testing algorithm. ${ }^{13}$ The results are given as units of "just noticeable difference" (JND).

\section{Randomization}

Patients were randomized within 4 weeks of the screening assessments to receive VEGF or placebo based upon a 3:1 randomization ratio, initially planned to be stratified by the presence or absence of symptomatic macrovascular disease of the legs (see protocol in supplementary material) but so few patients fell into the former group that the protocol was amended after the first 6 patients to omit stratification.

\section{Study agent}

Two VEGF agents were employed in this study. Pre-clinical data indicated bioequivalency of therapeutic effect using either VEGF-1/VEGF-A or VEGF-2/VEGF-C. The initial cohort of 16 patients was treated with VEGF-1 which was manufactured at St. Elizabeth's Medical Center, thereby limiting the application to a single center. Due to interest in moving forward into larger scale studies a change was made to VEGF-2 which was developed as a therapeutic agent by a commercial entity that manufactures clinical grade material suitable for distribution to multiple sites. At the completion of the first dose cohort $(2 \mathrm{mg}$ ) evidence of toxicity and all patient data relevant to adverse events was reviewed by the DSMB and an endorsement was made to continue and escalate the dose to $4 \mathrm{mg}$ (Fig. 1). The preparation of the plasmid is given in appendix D. Placebo injections were $0.9 \%$ sterile saline in $2.5 \mathrm{ml}$, the same volume as used for the active agent.

The active agent or placebo was divided and delivered to the patient in 8 intramuscular injections into the hamstring ( 2 injections spaced one-third and two-thirds of the distance from the buttock fold to the mid-popiliteal space); the meridian of the gastrocnemius ( 3 injections spaced one-, two- and three-quarters of the distance between the malleoli and the popiliteal fossa); and the meridian of the tibialis anterior ( 3 injections spaced one, two-, and three-quarters of the distance between the malleoli and the bottom of the patella). A second and a third series of 8 injections was administered at 2 and 4 weeks after the first treatment. The doses were developed based on animal data showing that reasonable levels of gene 
expression is limited to 2 weeks, while gene expression at 3 weeks is diminished and by 4 weeks is altogether absent. Three doses at intervals of 2 weeks were thus designed to sustain gene expression for approximately 6 weeks.

\section{Outcomes and Statistical Analysis}

The prespecified primary outcome was change in symptom score at 6 months in the treated leg. The protocol listed as a co-primary outcome the proportion of patients achieving 2 of the following: a) $\geq 2$ point decrease in SS; b) $\geq 4$ point decrease in TES; c) $\geq 30 \%$ improvement in peroneal or d) tibial nerve amplitude or e) summed motor amplitude; or f) $>30 \%$ improvement in sural nerve amplitude, if present at baseline. The secondary outcomes were differences changes at 6 months between the treated and untreated legs in the symptoms, examination and nerve conduction values.

Because of the small cohort sizes, VEGF-1 and VEGF-2 results and those with and without macrovascular disease were analyzed as one group.

Treatment group means of clinical and JND variables at baseline and six months were compared by the two-sample $t$ test. For these variables, the pre-specified method for analyzing changes from baseline to six months between treatment groups was an analysis of covariance (ANCOVA) with change in the treated leg as the outcome and change in the untreated leg as a continuous covariate. This method was chosen prior to opening the data set and performing statistical analysis in order to improve the precision of estimation of treatment effects. Because values of 0 were common in the electrophysiologic variables, Wilcoxon rank sum tests were used to compare the distributions of the baseline, six-month, and change measurements for these variables.

\section{Results}

A total of 1262 patients inquired about the study and 568 were screened by interview and review of records by one study nurse. Patients were excluded for the following reasons: complex medical history, blood chemistry abnormalities in record or prior amputations, 209; not diabetic, 115 or not on diabetic medications, 9 ; geographic constraints, 88 ; history of cancer, 58; active proliferative retinopathy, 21; penicillin allergy, 18 (Fig. 1). Thirty-nine patients were randomized to the active agent (16 to VEGF-1 and 23 to VEGF-2) and 11 to placebo. All protocol clinical and nerve conduction studies were completed with the exception of 3 patients who did not have full data from quantitative sensory studies.

Baseline clinical characteristics of the active and placebo groups are shown in Table 1. There were more males and more patients with insulin requirement in the VEGF group. HgbA1c values were similar. The initial symptom and examination scores and nerve conduction values are shown in Table 2 . There were no significant differences in the clinical or neurologic features but symptom scores (SS) and total examination scores (TES) tended to be higher (worse) in the treated legs of VEGF patients relative to placebo treated patients. There was a difference in the mean JND sensitivity to cold in the treated leg. Two placebo patients received a score of 0 in the treated leg at baseline and two had missing data. 


\section{Six-month absolute measures (Supplementary Table 1A)}

At 6 months, mean absolute symptom scores were lower in the placebo group for both the treated and untreated legs, as they were at baseline, with the difference achieving statistical significance in the untreated leg at 6 months (4.64 vs. 6.61; $\mathrm{p}=0.04)$. Total examination score was also lower (better) in the placebo group but none of the absolute differences between treatment groups achieved statistical significance. The median peroneal nerve amplitude was higher in the injected leg of placebo-group subjects than in the treated leg ( 2.6 vs. $0.6 \mathrm{mV} ; \mathrm{P}=0.1$ ). However, the peroneal amplitude was higher in the treated leg of VEGF patients than in the untreated leg ( 0.6 vs. 0.3 ; supplementary Table $1 \mathrm{~B})$. None of these amplitudes were significantly different from baseline.

HgbA1c rose slightly in both groups with a greater elevation in the placebo group $(0.15$ VEGF vs. 0.76 placebo).

\section{Six month outcomes (Table 3)}

The primary and secondary outcomes of changes between baseline and 6 months are shown in Tables 3A (clinical) and 3B (nerve conduction and quantitative sensory testing). A greater decline in clinical measures in Table 3 indicates a better outcome in relation to the comparator group. No significant differences between treatment groups were observed for the untreated leg. For comparisons in the treated leg, change in the untreated leg was used as a covariate. This resulted in substantially better precision. The 6 month change in symptom score was significantly different between treatment groups. Though the unadjusted changes in the treated leg were similar in the two treatment groups, the decline in mean symptom score in the untreated leg was larger for placebo-treated patients $(-1.91)$ than for VEGFtreated patients $(-0.74)$. Because change in the untreated leg was a strong predictor of change in the treated leg, the adjusted estimate of the difference between groups in change in the treated leg was -1.40 (s.e. $=0.52, \mathrm{P}=0.01)$.

Six of 39 patients in the treated group vs. 8 of 11 in the placebo group met the prespecified co-primary outcome of change in at least two of the five primary categories. No patient in either group showed greater than $30 \%$ increase in sural nerve action potential.

Among the secondary outcomes, comparing the active treatment leg to placebo, changes in the visual analog pain scale scores and distribution of pinprick loss favored the treatment group but changes in the other examination subscores (Table 3C), nerve conduction studies and quantitative sensory measures (Table 3B) were unchanged when adjusted for changes in the untreated leg.

\section{Adverse events}

Adverse events over 52 weeks are tabulated in Table 4A. Ten patients in the active treatment group had 22 serious adverse events (Table 4B); the placebo group had two serious adverse events in unique patients (chi-square for patients affected one-tailed p-value $=0.47$ ). No instances of worsening of active proliferative retinopathy were found. Increased claudication, diabetic foot infections or amputations occurred in the contralateral (untreated) limb. Overall there were 84 adverse events in the 39 treated patients and 51 events in the 11 
placebo patients. There were no deaths in the 52 weeks following enrollment and no hospitalizations related to the trial or to the study agent or the injections.

\section{Discussion}

The primary outcome of symptom score and secondary outcomes of visual pain scores distribution of sensory loss improved in the VEGF treated leg in comparison to the placebo treated leg and compared to the opposite (untreated) leg. Parallel improvement in these measures is plausible and is corroborated by reduced distribution of pinprick loss in the treated patients. The co-primary outcome of change in at least two of five categorical measures that included clinical and electrophysiologic change favored the treated group (12 of 39 vs. 2 of 11). Other measures, including nerve conduction studies, showed no statistically significant change. The improvement in the treated leg of one or two points on a summed symptom scale of 15 has clinical significance. As most of the motor and sensory nerve electrophysiologic measures were moderately to severely abnormal or absent on study entry, it is unlikely that a regenerative effect could have restored enough function to achieve measurable change in electrical function over a six month period. The lack of change in nerve conduction measures may have been related to the extent of electrophysiologic abnormality at baseline.

It is unlikely that the levels of circulating VEGF were adequate to explain the above findings. ${ }^{14}$ Hemoglobin A1c concentrations did not differ significantly between groups at any point and it is not plausible that any improvement in glycemic control in an individual patient affected one limb preferentially. An alternative interpretation of the improved sensory and pain scores was that VEGF worsened nerve function and thereby reduced symptoms. This is possible but unlikely in view of the parallel improvement in the distribution of sensory loss.

The adjustment for covariance was prespecified and chosen in order to improve precision of estimated treatment effect in a small study. Presumably by chance, placebo treated legs showed a larger decline in symptom score. As a result, ANCOVA both increased the estimated treatment effect and reduced its standard error, resulting in a significant treatment effect. This method and the improvement in the untreated leg, while valid and specified before analysis of the data set, temper any conclusions.

We used VEGF on the basis of its potential to promote neovascularization of the microcirculation of peripheral nerves although it may also have neurotrophic activity. Both isoforms of VEGF have been shown to be bioequivalent. As VEGF plays an important role in the pathogenesis of diabetic retinopathy and poses a theoretical risk of promoting neovascularization within occult tumors, we chose a delivery method designed to deliver the gene to the target organ without generating high circulating blood levels of the factor. This was accomplished by injecting naked plasmid DNA adjacent to the main nerve trunks of the leg with the aim of promoting VEGF expression in nerve. As in a previous study, ${ }^{5}$ we established that circulating VEGF above baseline levels could not be detected after these injections and that diabetic retinopathy did not worsen through the period of the study. There were also no instances of neoplastic disease in the year after injections. Mild leg swelling 
was observed in the treated leg of a few patients and it is not possible to determine if this led to unblinding.

The weaknesses of this study are its small sample size, the two isoforms and dose escalation of VEGF and the use of a non-standard scale for grading diabetic neuropathy. We have used this scale in a previous study ${ }^{5}$ and chose it in preference to other scales because of its simplicity and ease of administration, focus on symptoms and signs in the legs, and its ability to show therapeutic effect in a prior study. During our prior studies, several other scales and subscores failed to show changes even with gross alterations in foot and leg symptoms. The scale derived by Notermans and colleagues, from which our measures were derived, are discussed in the Methods section. However, the scale used in our study has not been validated against others.

Growth factor therapy for diabetic neuropathy had been attempted before in the form of nerve growth factor protein injected subcutaneously, three times per week for 48 weeks by Apfel and colleagues. ${ }^{1516}$ Their goal was to create a sustained circulating level of the protein that would act as a trophic factor for damaged nerves. Their initial trial with this approach yielded positive results, or at least a signal for benefit, but a larger randomized trial by the same investigators showed no effect. An analysis of the promising results of the two phase II trials and failure of the single phase III trial concluded that a "robust placebo effect, inadequate dosage, different study populations, and changes to the formulation of rhNGF for the phase III trial" may have explained the lack of benefit of growth factor therapy. ${ }^{17} \mathrm{~A}$ similar trial with subcutaneous injections of recombinant brain derived neurotrophic factor also failed to show an effect. ${ }^{18}$ All of these issues also apply to our study. In addition, we studied a severely affected group of patients with marked sensory symptoms and signs, and absence or marked reduction of motor and sensory nerve conduction potentials, thus limiting the chances for regeneration or improved nerve function. However, our preclinical studies suggested that the delivery of a trophic factor in physical proximity to nerve trunks had a likelihood of success and we designed our clinical trial based on these models.

Our study provides evidence that intramuscular VEGF gene therapy may improve symptoms of diabetic polyneuropathy and support a larger study to determine the therapeutic effects and safety of VEGF gene transfer in diabetic neuropathy.

\section{Supplementary Material}

Refer to Web version on PubMed Central for supplementary material.

\section{Acknowledgement}

We wish to recognize the crucial role played by Dr. Jeffrey Isner (deceased) in the experimental work, writing of the NIH grant and planning of this trial.

Funding: Supported by NIH Grant PO 1 HL66957-01A1, Dr. Ropper, P.I.; Dr. Douglas Losordo, Program Project P.I. 


\section{References}

1. Dyck PJ, Kratz KM, Lehman KA, et al. The prevalence by staged severity of various types of diabetic neuropathy, retinopathy, and nephropathy in a population-based cohort: the Rochester Diabetic Neuropathy Study. Neurology. 1993; 43:817-824. [PubMed: 8469345]

2. Schratzberger P, Walter D, Rittig K, et al. Reversal of experimental diabetic neuropathy by VEGF gene transfer. J Clin Invest. 2001; 107:1083-1092. [PubMed: 11342572]

3. Price SA, Dent C, Duran-Jimenez B, et al. Gene transfer of an engineered transcription factor promoting expression of VEGF-A protects against experimental diabetic neuropathy. Diabetes. 2006; 55:1847-1854. [PubMed: 16731852]

4. Murakami T, Arai M, Sunada Y, Nakamura A. VEGF 164 gene transfer by electroporation improves diabetic sensory neuropathy in mice. J Gene Med. 2006; 8:773-778. [PubMed: 16518878]

5. Simovic D, Isner JM, Ropper AH, Pieczek A, Weinberg DH. Improvement in chronic ischemic neuropathy after intramuscular phVEGF165 gene transfer in patients with critical limb ischemia. Arch Neurol. 2001; 58:761-768. [PubMed: 11346371]

6. Takeshita S, Losordo DW, Kearney M, et al. Time course of recombinant protein secretion after liposome-mediated gene transfer in a rabbit arterial organ culture model. Lab Invest. 1994; 71:387391. [PubMed: 7523763]

7. Losordo D, Pickering JG, Takeshita S, et al. Use of the rabbit ear artery to serially assess foreign protein secretion after site-specific arterial gene transfer in vivo. Circulation. 1994; 89:785-792. [PubMed: 8313567]

8. Brill V. NIS-LL: The primary measurement scale for clinical trial endpoints in diabetic neuropathy. Eur Neurol. 1999; 41(suppl 1):8-13. [PubMed: 10023123]

9. Merkies IS, Schmitz PI, van der Meché FG, et al. Psychometric evaluation of a new sensory scale in immune-mediated polyneuropathies. Inflammatory Neuropathy Cause and Treatment (INCAT) Group. Neurology. 2000; 54:943-949. [PubMed: 10690990]

10. Mendell JR, Barohn RJ, Freimer ML, et al. Working Group on Peripheral Neuropathy. Randomized controlled trial of IVIg in untreated chronic inflammatory demyelinating polyradiculoneuropathy. Neurology. 2001; 56:445-449. [PubMed: 11222785]

11. Bastyr EJ, Price KL, Bril V for the MBBQ Study Group. Development and Validity Testing of the Neuropathy Total Symptom Score-6: Questionnaire for the Study of Sensory Symptoms of Diabetic Peripheral Neuropathy. Clin Ther. 2005; 27:1278-1294. [PubMed: 16199253]

12. Notermans NC, Wokke JHJ, Lokhorst HM, Franssen H, Van der Graaf Y, Jennekens FGI. Polyneuropathy associated with monoclonal gammopathy of undetermined significance: a prospective study of the prognostic value of clinical and laboratory abnormalities. Brain. 1994; 117:1385-1393. [PubMed: 7820574]

13. Dyck PJ, O'Brien OC, Kosanke JL, et al. A 4, 2, and 1 stepping algorithm for quick and accurate estimation of cutaneous sensation threshold. Neurology. 1993; 43:1508-1512. [PubMed: 8351003]

14. Freedman SB, Vale P, Kalka C, et al. Plasma vascular endothelial growth factor (VEGF) levels after intramuscular and intramyocardial gene transfer of VEGF-1 plasmid DNA. Hum Gene Ther. 2002; 13:1595-1603. [PubMed: 12228014]

15. Apfel SC, Schwartz S, Adornato BT, et al. Efficacy and safety of recombinant human nerve growth factor in patients with diabetic polyneuropathy. JAMA. 2000; 284:2215-2221. [PubMed: 11056593]

16. Apfel SC, Kessler JA, Adornato BT, et al. Recombinant human nerve growth factor in the treatment of diabetic polyneuropathy. Neurology. 1998; 51:695-702. [PubMed: 9748012]

17. Apfel SC. Nerve growth factor for the treatment of diabetic neuropathy: what went wrong, what went right, and what does the future hold? Inter Rev Neurobiol. 2002; 50:393-413.

18. Wellmer A, Misra VP, Sharief MK, Kopelman PG, Anand P. A double-blind placebo-controlled clinical trial of recombinant human brain-derived neurotrophic factor (rhBDNF) in diabetic polyneuropathy. J Peripher Nerv Syst. 2001; 6:204-210. [PubMed: 11800042] 


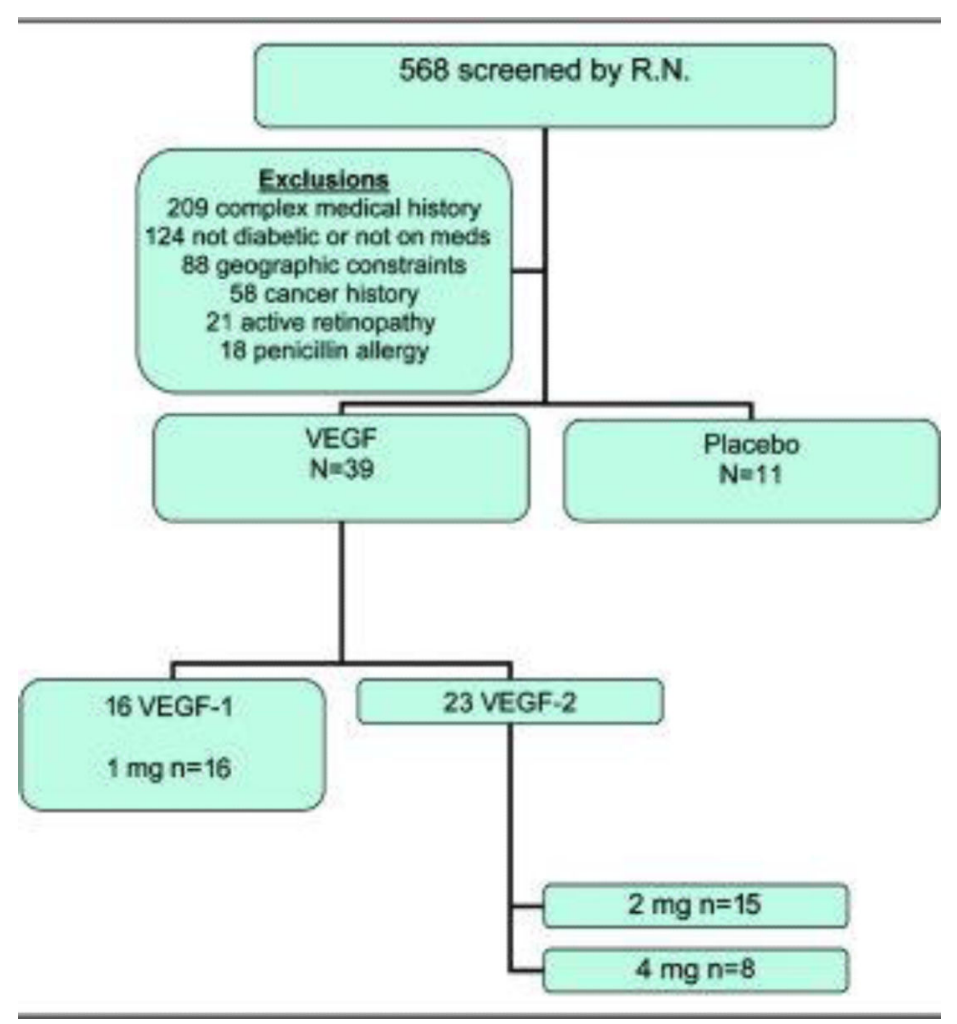

Figure 1.

Patient recruitment CONSORT diagram 


\section{Table 1}

\section{Baseline Clinical Features}

\begin{tabular}{lll} 
& VEGF $(\mathbf{n = 3 9})$ & Placebo $(\mathbf{n = 1 1})$ \\
\hline Age (Mean, s.d.) & $61.4+/-9.9$ & $65.6+/-8.4$ \\
Male & $18(46 \%)$ & $1(9 \%)$ \\
Diabetes Type I & $7(18 \%)$ & 0 \\
Diabetes Type II & $32(82 \%)$ & $11(100 \%)$ \\
Diabetes Duration (yrs) & $12.7+/-10.6$ & $13.2+/-7.4$ \\
Insulin use & $17(44 \%)$ & $7(64 \%)$ \\
Hgb A1C baseline & $7.6+/-1.4$ & $7.6+/-2$ \\
\hline
\end{tabular}


Table 2

Baseline Scores and Electrophysiologic Values, By Leg and Treatment Group (Mean, s.d.)

\begin{tabular}{|c|c|c|c|}
\hline & VEGF $n=39$ & Placebo $n=11$ & $P$ value \\
\hline \multicolumn{4}{|l|}{ Symptom Score } \\
\hline Untreated Leg & $7.26(2.46)$ & $6.55(2.50)$ & 0.40 \\
\hline Treated Leg & $7.62(2.57)$ & $6.18(1.54)$ & 0.09 \\
\hline \multicolumn{4}{|c|}{ Sensory Examination Score } \\
\hline Untreated Leg & $20.10(5.28)$ & $17.55(6.59)$ & 0.19 \\
\hline Treated Leg & $20.33(5.32)$ & $17.55(5.47)$ & 0.13 \\
\hline \multicolumn{4}{|l|}{ Motor Score } \\
\hline Untreated Leg & $2.82(3.89)$ & $2.55(3.27)$ & 0.83 \\
\hline Treated Leg & $2.92(4.04)$ & $2.09(3.24)$ & 0.53 \\
\hline \multicolumn{4}{|l|}{ Reflex Score } \\
\hline Untreated Leg & $5.64(2.19)$ & $4.36(1.75)$ & 0.08 \\
\hline Treated Leg & $5.44(2.10)$ & $4.18(1.40)$ & 0.07 \\
\hline \multicolumn{4}{|l|}{ Total Exam Score } \\
\hline Untreated Leg & $28.56(8.43)$ & $24.45(8.68)$ & 0.16 \\
\hline Treated Leg & $28.69(8.52)$ & $23.82(8.41)$ & 0.10 \\
\hline \multicolumn{4}{|l|}{ VAS Pain Score } \\
\hline Untreated Leg & $5.15(2.94)$ & $4.45(2.11)$ & 0.47 \\
\hline Treated Leg & $5.44(3.08)$ & $4.45(2.54)$ & 0.34 \\
\hline \multicolumn{4}{|c|}{ Electrophysiologic Variables (Median, Interquartile range) } \\
\hline \multicolumn{4}{|c|}{ Tibial Amplitude (mV) } \\
\hline Untreated Leg & $0.3(0.0,3.6)$ & $0.9(0.1,9.2)$ & 0.32 \\
\hline Treated Leg & $0.4(0.0,2.5)$ & $3.7(0.1,8.3)$ & 0.15 \\
\hline \multicolumn{4}{|c|}{ Peroneal Amplitude (mV) } \\
\hline Untreated Leg & $0.4(0.0,1.9)$ & $2.8(0.1,4.3)$ & 0.10 \\
\hline Treated Leg & $0.6(0.0,2.4)$ & $1.8(0.2,3.5)$ & 0.21 \\
\hline \multicolumn{4}{|c|}{ Amplitude Sum (mV) } \\
\hline Untreated Leg & $0.6(0.2,4.8)$ & $3.1(0.2,15.1)$ & 0.26 \\
\hline Treated Leg & $1.1(0.1,6.4)$ & $4.8(0.3,13.3)$ & 0.19 \\
\hline \multicolumn{4}{|c|}{ Sural Amplitude $(\mu \mathrm{V})$} \\
\hline Untreated Leg & $0.0(0.0,0.0)$ & $0.0(0.0,2.7)$ & 0.51 \\
\hline Treated Leg & $0.0(0.0,1.7)$ & $1.5(0.0,4.4)$ & 0.22 \\
\hline \multicolumn{4}{|c|}{ JND Variables (Mean, Standard Deviation) } \\
\hline \multicolumn{4}{|l|}{ Cold } \\
\hline Untreated Leg & $19.31(6.64)$ & $17.74(5.04)$ & 0.51 \\
\hline Treated Leg & $20.97(5.40)$ & $15.19(9.19)$ & 0.02 \\
\hline \multicolumn{4}{|l|}{ Vibration } \\
\hline Untreated Leg & $22.80(5.01)$ & $23.48(1.97)$ & 0.69 \\
\hline
\end{tabular}




\begin{tabular}{|l|c|c|c|}
\hline & VEGF $\mathbf{n = 3 9}$ & Placebo $n=11$ & P value \\
\hline Treated Leg & $22.81(4.60)$ & $23.13(1.63)$ & 0.84 \\
\hline
\end{tabular}


Table 3A

Changes in Clinical Measures Between Baseline and Six Months, by Leg and Treatment Group

\begin{tabular}{|c|c|c|c|}
\hline Treatment Group & VEGF $(n=39)$ & Placebo $(n=11)$ & P value* \\
\hline \multicolumn{4}{|c|}{ Clinical Variable (Mean, Standard Error) } \\
\hline \multicolumn{4}{|l|}{ Symptom Score ${ }^{+}$} \\
\hline Untreated Leg & $-0.74(0.50)$ & $-1.91(0.59)$ & 0.24 \\
\hline Treated Leg & $-1.21(0.53)$ & $-0.91(0.65)$ & 0.01 \\
\hline \multicolumn{4}{|c|}{ Sensory Examination Score } \\
\hline Untreated Leg & $-0.42(0.64)$ & $-1.18(1.55)$ & 0.60 \\
\hline Treated Leg & $-0.89(0.57)$ & $-1.18(1.43)$ & 0.71 \\
\hline \multicolumn{4}{|l|}{ Motor Score } \\
\hline Untreated Leg & $-0.18(0.36)$ & $-0.09(0.49)$ & 0.90 \\
\hline Treated Leg & $-0.39(0.34)$ & $0.09(0.41)$ & 0.36 \\
\hline \multicolumn{4}{|l|}{ Reflex Score } \\
\hline Untreated Leg & $-0.05(0.30)$ & $0.73(0.49)$ & 0.21 \\
\hline Treated Leg & $0.16(0.30)$ & $0.91(0.41)$ & 0.72 \\
\hline \multicolumn{4}{|l|}{ Total Exam Score } \\
\hline Untreated Leg & $-0.66(0.83)$ & $-0.55(1.61)$ & 0.95 \\
\hline Treated Leg & $-1.13(0.75)$ & $-0.18(1.66)$ & 0.37 \\
\hline \multicolumn{4}{|l|}{ VAS Pain Score } \\
\hline Untreated Leg & $-0.92(0.42)$ & $-1.45(0.82)$ & 0.56 \\
\hline Treated Leg & $-1.47(0.48)$ & $-0.45(1.11)$ & 0.01 \\
\hline
\end{tabular}

* $\mathrm{P}$ values for treated leg obtained from analysis of covariance adjusting for change in the untreated leg

${ }^{+}$Primary outcome 
Table 3B

Changes in Electrophysiologic and JND Variables Between Baseline and Six Months, by Leg and Treatment Group

\begin{tabular}{|c|c|c|c|}
\hline Treatment Group & VEGF & Placebo & P value \\
\hline Changes in Electrophysiologic Variables (Median, Interquartile Range $)$ \\
\hline Tibial Amplitude & & & \\
\hline Untreated Leg & $0.0(-0.3,0.1)$ & $-0.1(-1.3,0.0)$ & 0.33 \\
\hline Treated Leg & $0.0(-0.2,0.1)$ & $-0.4(-2.3,0.0)$ & 0.27 \\
\hline Peroneal Amplitude & & & \\
\hline Untreated Leg & $0.0(-0.2,0.1)$ & $0.0(-0.3,0.6)$ & 0.88 \\
\hline Treated Leg & $0.0(-0.3,0.0)$ & $0.0(-0.1,0.9)$ & 0.12 \\
\hline Amplitude Sum & & & \\
\hline Untreated Leg & $0.0(-0.3,0.1)$ & $0.0(-3.0,0.4)$ & 0.80 \\
\hline Treated Leg & $-0.1(-0.4,0.0)$ & $0.0(-1.0,0.1)$ & 0.64 \\
\hline Sural Amplitude & & & \\
\hline Untreated Leg & $0.0(0.0,0.0)$ & $0.0(-0.5,0.0)$ & 0.44 \\
\hline Treated Leg & $0.0(0.0,0.0)$ & $0.0(-0.8,0.0)$ & 0.36 \\
\hline Changes in JND Measurements (Mean, Standard Error) \\
\hline Cold & & & \\
\hline Untreated Leg & $-0.15(1.11)$ & $-3.14(3.98)$ & 0.31 \\
\hline Treated Leg & $-0.68(1.21)$ & $2.01(4.65)$ & 0.30 \\
\hline Vibration & & & \\
\hline Untreated Leg & $0.84(0.81)$ & $-1.66(1.31)$ & 0.15 \\
\hline Treated Leg & $0.07(0.88)$ & $-0.43(0.51)$ & 0.69 \\
\hline
\end{tabular}


Table 3C

Changes in SES Subscores between Baseline and Six Months by Leg and Treatment Group

\begin{tabular}{|c|c|c|l|}
\hline Treatment Group & VEGF & Placebo & P value \\
\hline \multicolumn{3}{|l|}{${ }^{*}$} \\
\hline SES Subscore (Mean, Standard Error) & & \\
\hline Sensation - Pin & & & \\
\hline Untreated Leg & $-0.11(0.15)$ & $-1.09(0.48)$ & $\mathbf{0 . 0 1}$ \\
\hline Treated Leg & $-0.21(0.18)$ & $-0.64(0.45)$ & 0.15 \\
\hline Sensation - Touch & & & \\
\hline Untreated Leg & $0.24(0.20)$ & $0.27(0.75)$ & 0.95 \\
\hline Treated Leg & $0.11(0.22)$ & $0.36(0.69)$ & 0.42 \\
\hline Distribution - Pin & & & \\
\hline Untreated Leg & $-0.18(0.14)$ & $-0.27(0.24)$ & 0.76 \\
\hline Treated Leg & $-0.26(0.12)$ & $0.18(0.18)$ & $\mathbf{0 . 0 1 7}$ \\
\hline Distribution - Touch & & & \\
\hline Untreated Leg & $-0.13(0.14)$ & $0.00(0.40)$ & 0.70 \\
\hline Treated Leg & $-0.16(0.15)$ & $-0.09(0.34)$ & 0.92 \\
\hline Vibration - Toe & & & \\
\hline Untreated Leg & $-0.16(0.15)$ & $-.73(0.33)$ & 0.10 \\
\hline Treated Leg & $-0.32(0.15)$ & $-.73(0.36)$ & 0.70 \\
\hline Vibration - Ankle & & & \\
\hline Untreated Leg & $-0.16(0.19)$ & $-0.09(0.25)$ & 0.23 \\
\hline Treated Leg & $-0.11(0.19)$ & $-0.09(0.25)$ & 0.47 \\
\hline Proprioception - Toe & & & \\
\hline Untreated Leg & $0.08(0.25)$ & $-0.18(0.46)$ & 0.62 \\
\hline Treated Leg & $0.05(0.20)$ & $-0.18(0.46)$ & 0.60 \\
\hline
\end{tabular}

P values for treated leg obtained from analysis of covariance adjusting for change in the untreated leg.

Assuming equal variances. The $\mathrm{P}$ value assuming unequal variances is 0.07 . 
Table 4A

All Adverse Events Over 52 Weeks ${ }^{*}$

\begin{tabular}{llll}
\hline & $\begin{array}{l}\text { Placebo }(\mathbf{n}=\mathbf{1 1}) \text { Events/Sub } \\
(\boldsymbol{\%} \text { Sub) }\end{array}$ & $\begin{array}{l}\text { Treatment }(\mathbf{n}=\mathbf{3 9}) \text { Events/Sub } \\
(\boldsymbol{\%} \text { Sub) }\end{array}$ & $\begin{array}{l}\text { Total (n=50) Events/Subj } \\
(\boldsymbol{\%} \text { Sub) }\end{array}$ \\
\cline { 2 - 4 } Chest Pain & $2 / 1(9.1)$ & $2 / 2(5.1)$ & $4 / 3(6.0)$ \\
Diabetic Retinopathy & $2 / 2(18.2)$ & $4 / 3(7.7)$ & $6 / 5(10.0)$ \\
Ecchymosis & $2 / 1(9.1)$ & $0 / 0(0.0)$ & $2 / 1(2.0)$ \\
Epistaxis & $0 / 0(0.0)$ & $6 / 1(2.6)$ & $6 / 1(2.0)$ \\
Excoriation & $0 / 0(0.0)$ & $7 / 5(12.8)$ & $7 / 5((10.0)$ \\
Eye Arterial Narrowing & $2 / 1(9.1)$ & $0 / 0(0.0)$ & $2 / 1(2.0)$ \\
Eye Hemorrhage & $3 / 2(18.2)$ & $0 / 0(0.0)$ & $3 / 2(4.0)$ \\
Eye Vascular Periphery Narrowing & $2 / 1(9.1)$ & $0 / 0(0.0)$ & $2 / 1(2.0)$ \\
Hemoglobin Decreased & $2 / 2(18.2)$ & $1 / 1(2.6)$ & $3 / 3(6.0)$ \\
Hematocrit Decreased & $2 / 2(18.2)$ & $0 / 0(0.0)$ & $2 / 2(4.0)$ \\
Macular Degeneration & $2 / 1(9.1)$ & $0 / 0(0.0)$ & $2 / 1(2.0)$ \\
Muscle Cramp & $0 / 0(0.0)$ & $6 / 3(7.7)$ & $6 / 3(6.0)$ \\
Pain & $5 / 2(18.2)$ & $4 / 2(5.1)$ & $9 / 4(8.0)$ \\
Pain in Extremity (20.0) & $5 / 3(27.3)$ & $11 / 7(17.9)$ & $16 / 10$ \\
Peripheral Edema (40.0) & $13 / 5(45.4)$ & $35 / 15(38.5)$ & $48 / 20$ \\
Rectal Hemorrhage & $0 / 0(0.0)$ & $6 / 3(7.7)$ & $6 / 3(6.0)$ \\
Red Blood Cell Count Decreased & $2 / 2(18.2)$ & $0 / 0(0.0)$ & $2 / 2(4.0)$ \\
Retinal Exudates & $3 / 1(9.1)$ & $0 / 0(0.0)$ & $3 / 1(2.0)$ \\
Urinary Tract Infection & $2 / 2(18.2)$ & $2 / 2(5.1)$ & $4 / 4(8.0)$ \\
Vertigo & $2 / 1(9.1)$ & $0 / 0(0.0)$ & $2 / 1(2.0)$ \\
\hline
\end{tabular}

* Subjects could have more than one adverse event.

$$
\text { * }
$$

*Adverse events were included if they occurred in at least $15 \%$ of either group. 
Table 4B

Serious Adverse Events (SAE) Over 52 Weeks

\begin{tabular}{ll}
\hline Treatment Group: & 10 subjects \\
\hline Subject Number & SAE \\
\hline $01-1102$ & 2 episodes of myocardial ischemia \\
$001-1103$ & 3 episodes of congestive heart failure \\
& 1 episode of worsening of vascular disease with gangrene \\
$01-1108$ & 2 episodes of calf claudication \\
& 2 episodes of carotid artery disease \\
& 1 episode of coronary artery disease \\
$01-1113$ & 2 episodes of severe asthma \\
$001-2102$ & 1 episode of colorectal bleeding \\
$001-2106$ & 2 episodes of diabetic foot infection \\
$001-2109$ & 1 episode of worsening depression \\
$001-2110$ & 1 episode of CHF \\
$001-2115$ & 1 episode of myocardial infarction \\
& 1 episode of stroke \\
\hline $001-2116$ & 2 subjects \\
\hline Placebo Group: & SAEs Reported \\
\hline Subject Number & episode of Left Foot Trauma-cellulitis \\
\hline $01-1121$ &
\end{tabular}

